\title{
Rapid response to mycophenolate mofetil in combination with romiplostim in a case of severe refractory immune thrombocytopenia post COVID-19 vaccination
}

\author{
Snigdha Nutalapati $\odot ｜$ Garima Gupta | Gerhard C. Hildebrandt
}

Division of Hematology and Oncology, University of Kentucky College of Medicine, Lexington, Kentucky, USA

\section{Correspondence}

Snigdha Nutalapati, Department of Hematology and Oncology, University of Kentucky, Markey Cancer Center CC402, 800 Rose Street, Lexington, KY 40536-0093, USA.

Email: SNutalapati@uky.edu

\begin{abstract}
Vaccine mediated immune mediated thrombocytopenia (ITP) is exceedingly rare. We present a case of a young female who developed severe refractory ITP with multiple bleeding sites post second dose of COVID-19 vaccination. She was treated with a combination of romiplostim and mycophenolate mofetil that resulted in rapid platelet count recovery.
\end{abstract}

K E Y W O R D S

immune thrombocytopenia, mycophenolate mofetil, romiplostim, SARS-CoV2 vaccination

\section{1 | INTRODUCTION}

Immune-mediated thrombocytopenia (ITP) is an autoimmune condition characterized by low platelet counts and wide range of clinical manifestations from asymptomatic presentation to major bleeding manifestations. Vaccinemediated secondary ITP is rare and is associated with influenza, measles mumps rubella (MMR), and hepatitis A and $\mathrm{B}$ vaccination. Most patients respond to steroids and intravenous immunoglobulin (IVIG). ${ }^{1}$ We hereby report a case of a 26-year-old woman with severe refractory ITP who presented with multiple active bleeding sites leading to a significant drop in hemoglobin post second dose of mRNA1273 SARS-CoV-2 vaccine. She was refractory to treatment with steroids and IVIG and was eventually treated with combination of romiplostim and mycophenolate mofetil (MMF) with which her counts improved dramatically.

\section{CASE DISCUSSION}

A 25-year-old Caucasian woman with no significant past medical history except for well-controlled bronchial asthma presented to the hospital with two-day history of worsening petechiae that initially started on her face and lower extremities and eventually spread to her back, chest, abdomen, and upper extremities. She also complained of intermittent epistaxis.

Vital signs on initial presentation including blood pressure, heart rate, and oxygen saturation were normal. Physical examination revealed extensive bruising over her back, chest, and extremities along with scattered petechiae all over the body. Laboratory findings on presentation included WBC of $11 \times 10^{9} / \mathrm{L}$, hemoglobin $(\mathrm{Hb})$ of $11 \mathrm{~g} / \mathrm{dl}$, and severe thrombocytopenia with a platelet count of 1 $\times 10^{9} / \mathrm{L}$. Prothrombin time (PT/INR), activated partial thromboplastin time (aPTT), fibrinogen, lactate dehydrogenase (LDH), absolute reticulocyte count, and haptoglobin levels were within normal limits. Peripheral blood smear showed profound thrombocytopenia with no evidence of schistocytes. RT-PCR testing for SARS-CoV-2 infection was negative. Autoimmune and infectious workup including antinuclear antibody (ANA), coombs testing, HIV ELISA, and serology for hepatitis B and C were negative. Ultrasound of the abdomen did not show evidence of splenomegaly or liver parenchymal changes. 
Her most recent laboratory testing including $\mathrm{Hb}$ and platelet count from three months ago during routine primary care visit, was within normal limits. She neither reported personal or family history of bleeding disorders nor had required blood transfusions prior to this presentation. 26 days prior to this presentation, she had received her second dose of mRNA-1273 SARS-CoV-2 vaccine.

Given her clinical presentation, negative infectious and autoimmune workup, normal hemolysis and coagulation studies along with absence of other identifiable underlying etiology, she was diagnosed with ITP and was initiated on dexamethasone $40 \mathrm{mg}$ daily for four days along with IVIG $1 \mathrm{~g} / \mathrm{kg}$ for two days. She continued to have clinical worsening during the second and third day of hospitalization and developed multiple bleeding sites including gross hematuria, hematochezia, and subconjunctival hemorrhage. Her hemoglobin dropped from $11 \mathrm{~g} / \mathrm{dl}$ on presentation to $6.4 \mathrm{~g} / \mathrm{dl}$. She was initiated on tranexamic acid for bleeding control and supportive care with platelet transfusions. Her platelet counts continued to remain low $\left(5 \times 10^{9} / \mathrm{L}\right)$ despite receiving 4 days of dexamethasone, 2 days of IVIG, and platelet transfusion support. She continued to have intermittent epistaxis; however, hematuria and rectal bleeding started improving with tranexamic acid initiation.

As there was no improvement in platelet counts with IVIG and dexamethasone, on day 7, she was initiated on MMF $1 \mathrm{~g}$ twice daily and romiplostim $1 \mathrm{mcg} / \mathrm{kg}$ weekly. On day 12 , her platelet counts for the first time improved to $13 \times 10^{9} / \mathrm{L}$. Hb remained stable around $11 \mathrm{~g} / \mathrm{dl}$. She was discharged after receiving second dose of romiplostim 3 $\mathrm{mcg} / \mathrm{kg}$ on day 14. During her first outpatient visit on day 21 , her platelet counts improved to $140 \times 10^{9} / \mathrm{L}$. She went on to receive third dose of romiplostim at $3 \mathrm{mcg} / \mathrm{kg}$. She had no evidence of active bleeding. During fourth week follow-up, platelet count recovered to $268 \times 10^{9} / \mathrm{L}$ and MMF was tapered down to $500 \mathrm{mg}$ twice daily and romiplostim to $2 \mathrm{mcg} / \mathrm{kg}$. By fifth-week, her platelet counts continued to improve to $324 \times 10^{9} / \mathrm{L}$ and MMF was tapered to $250 \mathrm{mg}$ twice daily and romiplostim to $1 \mathrm{mcg} / \mathrm{kg}$. She continued to have normal and stable platelet counts, and eventually, MMF and romiplostim were discontinued at her sixth-week follow-up visit. She continued follow-up in the hematology clinic for 3 months from discharge from the hospital after which she was advised to resume care with her primary care physician.

\section{DISCUSSION}

ITP is an autoimmune entity characterized by autoantibodies directed against platelet antigens leading to platelet destruction and thrombocytopenia. Clinical manifestations range from asymptomatic presentation, spontaneous petechiae and purpura, mucocutaneous hemorrhage to fatal complications such as gastrointestinal bleeding or intracranial hemorrhage, which usually occur when platelet count is below $10 \times 10^{9} / \mathrm{L}$. $^{2}$

Vaccination as an etiology for secondary ITP was previously reported with influenza, hepatitis A and B, measles, MMR, and diphtheria tetanus acellular pertussis (DTaP) vaccines. It is hypothesized that pathogenesis of ITP is mainly secondary to molecular mimicry, a phenomenon in which host antigens are recognized as antigens of immunization, provoking the development of autoantibodies. These antibody-coated platelets may undergo reticuloendothelial phagocytosis resulting in reduced platelet survival. It is also proposed that vaccine-associated autoimmunity can stem not only from antigen-mediated responses but also contributed by other constituents of vaccine such as adjuvants, preservatives, or diluents. ${ }^{1,3}$

Lee et al. described 20 patients who developed thrombocytopenia after receiving COVID vaccination with either mRNA-1273 or Pfizer-BioNTech BNT16B2b2 mRNA SARS-CoV-2 vaccine. Sixteen of these patients did not report history of thrombocytopenia prior to their vaccination. ${ }^{4}$ Onset of symptoms from the date of vaccination ranged from 1 to 23 days. ${ }^{4}$ In the other three individually published case reports, ITP developed $12 \mathrm{~h}$ to 5 days post Pfizer-BioNTech BNT16B2b2 mRNA SARS-CoV-2 vaccine in patients who previously had a normal platelet count. ${ }^{5-7}$ Similarly, our patient had normal platelet counts prior to this presentation. ITP developed on day 26 after the second vaccine was given, which differs from previously reported cases and, so far, is the most "delayed" onset reported in the literature. It is not clear how the immunologic mimicry happens after SARS-CoV2 vaccination and what the immunologic target is. Immune responses to vaccines are highly variable between different individuals and populations, and there may be other factors implicated such as the microbiome impact on vaccine immunity. ${ }^{8}$ Corticosteroids, IVIG with or without platelet transfusions, have been used successfully with responses noted within 4-6 days. ${ }^{5-7}$ In the study by Lee et al, outcomes were reported in 16 patients, of which 13 responded to the initial treatment with IVIG, steroids, or platelet transfusion. One patient received rituximab, romiplostim, vincristine along with steroids and IVIG. Another patient developed severe thrombocytopenia and intracranial hemorrhage despite receiving corticosteroids, IVIG, and Rituximab. ${ }^{4}$

In our case, thrombocytopenia was refractory to initial treatment with dexamethasone and IVIG. Due to her presentation with massive and multi-site bleeding, there was concern for imminent intracranial hemorrhage in 
this young and otherwise healthy female, and a rapid response to treatment of her refractory ITP seemed critical. We were hesitant to use commonly recommended options for refractory ITP, such as rituximab, single-agent thrombopoietin-stimulating agent (TSA), and spleen tyrosine kinase inhibitor fostamatinib due to acuity of this case. ${ }^{9}$ With rituximab, median time to response is 5.5 weeks, ${ }^{10}$ and TSAs and fostamatinib are approved for patients with chronic ITP who have failed conventional medical therapy or splenectomy. ${ }^{11-13}$ Although promising, there are limited data to support the use of single-agent TSAs in newly diagnosed ITP. ${ }^{14}$ In a pooled analysis of phase III clinical trials studying fostamatinib in patients with chronic ITP, only $18 \%$ of patients achieved a stable response. ${ }^{15}$ Splenectomy was prohibited due to bleeding risk and would increase lifelong susceptibility to encapsulated bacteria. ${ }^{9}$

Promising activity for MMF in the treatment of de novo and refractory ITP has emerged over the recent years, and after careful consideration and extensive discussion with the patient and her family, we decided to combine MMF with the TSA romiplostim. The combination of immunosuppressants with IVIG and TSA has been reported in a small series to be efficacious and safe. ${ }^{16,17}$ The response to this combination was rapid and vigorous.

As SARS-CoV-2 pandemic persists, concerns for vaccine-related side effects remain, both among caregivers and the general population. As of June 26, 2021, around $46.4 \%$ of the United States population has received full vaccination against SARS-CoV-2 and the risks of serious adverse events remain extremely low. Similar to primary ITP, vaccine-related ITP can have a broad spectrum of presentation ranging from mild bleeding and petechiae to fatal bleeding manifestations. When patients present with ITP, a thorough vaccination history is needed. ITP after SARS-CoV-2 vaccination is rare, and the potential association is often overlooked and missed. While neither of the clinical trials studying mRNA-1273 or Pfizer-BioNTech BNT16B2b2 mRNA SARS-CoV-2 vaccine had documented cases of thrombocytopenia,${ }^{18}$ it is worthwhile to note that possibility of reported thrombocytopenia postvaccination could be merely a coincidence rather than a causative factor. Nevertheless, we are probably just now only at the beginning of our understanding of the plethora and complexity of side effects and immunological impact of the SARS-CoV-2-directed vaccination and the virus itself. Continued vigilance and reporting of the possible side effects of vaccinations are needed, and we believe this should not preclude individuals from getting vaccinated given rarity of these events and the proven effectiveness of vaccine in mitigating this horrendous pandemic.

Further studies on the aggressive management with combination regimens including immunosuppressants and thrombopoietin agonists, especially among patients with refractory thrombocytopenia and bleeding complications, are warranted.

\section{CONFLICT OF INTEREST}

The authors report no conflict of interest.

\section{AUTHOR CONTRIBUTIONS}

Snigdha Nutalapati contributed to the case management and manuscript writing. Garima Gupta contributed to the case management and manuscript writing. Gerhard C. Hildebrandt contributed to the case management, manuscript writing, and final approval.

\section{CONSENT}

The authors confirm that the patient provided informed consent in accordance with the journal's patient consent policy.

\section{DATA AVAILABILITY STATEMENT}

Data sharing is not applicable as no new data were generated.

\section{ORCID}

Snigdha Nutalapati (D) https://orcid.

org/0000-0002-4753-7177

\section{REFERENCES}

1. Perricone C, Ceccarelli F, Nesher G, et al. Immune thrombocytopenic purpura (ITP) associated with vaccinations: a review of reported cases. Immunol Res. 2014;60(2-3):226-235. https://doi. org/10.1007/s12026-014-8597-x

2. Rodeghiero F, Stasi R, Gernsheimer T, et al. Standardization of terminology, definitions and outcome criteria in immune thrombocytopenic purpura of adults and children: report from an international working group. Blood. 2009;113(11):23862393. https://doi.org/10.1182/blood-2008-07-162503

3. Rinaldi M, Perricone C, Ortega-Hernandez O-D, Perricone R, Shoenfeld Y. Immune thrombocytopaenic purpura: an autoimmune cross-link between infections and vaccines. Lupus. 2014;23(6):554-567. https://doi.org/10.1177/0961203313499959

4. Lee EJ, Cines DB, Gernsheimer T, et al. Thrombocytopenia following Pfizer and Moderna SARS-CoV-2 vaccination. Am J Hematol. 2021;96(5):534-537. https://doi.org/10.1002/ ajh.26132

5. Fueyo-Rodriguez O, Valente-Acosta B, Jimenez-Soto R, et al. Secondary immune thrombocytopenia supposedly attributable to COVID-19 vaccination. BMJ Case Rep. 2021;14(5). https:// doi.org/10.1136/bcr-2021-242220

6. Pasin F, Calabrese A, Pelagatti L. Immune thrombocytopenia following COVID-19 mRNA vaccine: casuality or causality? Intern Emerg Med. 2021. https://doi.org/10.1007/s11739-02102778-w

7. Tarawneh O, Tarawneh H. Immune thrombocytopenia in a 22-year-old post Covid-19 vaccine. Am J Hematol. 2021;96(5):E1 33-E134. https://doi.org/10.1002/ajh.26106 
8. de Jong SE, Olin A, Pulendran B. The impact of the microbiome on immunity to vaccination in humans. Cell Host Microbe. 2020;28(2):169-179. https://doi.org/10.1016/j.chom. 2020.06.014

9. Cuker A, Prak ETL, Cines DB. Can immune thrombocytopenia be cured with medical therapy? Semin Thromb Hemost. 2015;41(4):395-404.

10. Arnold DM, Dentali F, Crowther MA, et al. Systematic review: efficacy and safety of rituximab for adults with idiopathic thrombocytopenic purpura. Ann Intern Med. 2007;146(1):2533. https://doi.org/10.7326/0003-4819-146-1-200701020-00006

11. Bussel JB, Arnold DM, Boxer MA, et al. Long-term fostamatinib treatment of adults with immune thrombocytopenia during the phase 3 clinical trial program. Am J Hematol. 2019;94(5):546553. https://doi.org/10.1002/ajh.25444

12. Bussel JB, Provan D, Shamsi T, et al. Effect of eltrombopag on platelet counts and bleeding during treatment of chronic idiopathic thrombocytopenic purpura: a randomised, doubleblind, placebo-controlled trial. Lancet. 2009;373(9664):641-648. https://doi.org/10.1016/S0140-6736(09)60402-5

13. Kuter DJ, Rummel M, Boccia R, et al. Romiplostim or standard of care in patients with immune thrombocytopenia. $N$ Engl J Med. 2010;363(20):1889-1899. https://doi.org/10.1056/NEJMo a1002625

14. González-López TJ, Fernández-Fuertes F, HernándezRivas JA, et al. Efficacy and safety of eltrombopag in persistent and newly diagnosed ITP in clinical practice. Int $J$ Hematol. 2017;106(4):508-516. https://doi.org/10.1007/s1218 5-017-2275-4
15. Connell NT, Berliner N. Fostamatinib for the treatment of chronic immune thrombocytopenia. Blood. 2019;133(19):20272030. https://doi.org/10.1182/blood-2018-11-852491

16. Gudbrandsdottir S, Leven E, Imahiyerobo A, Lee CS, Bussel J. Combination of thrombopoietin receptor agonists, immunosuppressants and intravenous immunoglobulin as treatment of severe refractory immune thrombocytopenia in adults and children. Br J Haematol. 2020;189(2):e37-e40. https://doi.org/ 10.1111/bjh.16426

17. Pell J, Greenwood R, Ingram J, et al. Trial protocol: a multicentre randomised trial of first-line treatment pathways for newly diagnosed immune thrombocytopenia: standard steroid treatment versus combined steroid and mycophenolate. The FLIGHT trial. BMJ Open. 2018;8(10):e024427. https://doi. org/10.1136/bmjopen-2018-024427

18. Walsh EE, Frenck RW, Falsey AR, et al. Safety and Immunogenicity of two RNA-based Covid-19 vaccine candidates. N Engl J Med. 2020;383(25):2439-2450. https://doi.org/ 10.1056/NEJMoa2027906

How to cite this article: Nutalapati S, Gupta G, Hildebrandt GC. Rapid response to mycophenolate mofetil in combination with romiplostim in a case of severe refractory immune thrombocytopenia post COVID-19 vaccination. Clin Case Rep. 2021;9:e05035. https://doi.org/10.1002/ccr3.5035 